\title{
含 1,3,4-噻二唑的二硫醚衍生物的合成及抗肿瘤活性研究
}

\author{
李莎 荆芬付小云 赵继军王雪峰 \\ 李宝林刘玉明陈宝泉* \\ (天津理工大学化学化工学院 天津 300384)
}

\begin{abstract}
摘要 以硫醇、硫脲、2-氨基-5-颈基-1,3,4-噻二唑等为原料, 通过两步反应合成了 10 个新的含 1,3,4-噻二唑的二硫醚 衍生物, 并利用 IR, ${ }^{1} \mathrm{H}$ NMR, ESI-MS 和元素分析对目标化合物进行了结构表征. 采用 CCK-8 法测试了目标产物对人 体肝癌细胞 SMMC-7721, 乳腺癌细胞 MCF-7 和肺癌细胞 A549 等肿瘤细胞的增殖抑制活性. 结果表明, 对于不同的肿 瘤细胞, 大多数试验化合物显示了较好的增殖抑制活性, 且其活性优于阳性对照药 5-氟尿嘧啶. 尤其是正丙基(2-氨基1,3,4-噻二唑-5-基)二硫醚(3b)和正丁基(2-氨基-1,3,4-噻二唑-5-基)二硫醚(3d), 对 SMMC-7721 细胞显示了高效的增殖抑 制效果, $\mathrm{IC}_{50}$ 值分别为 1.68 和 $1.93 \mu \mathrm{mol} / \mathrm{L}$. 4-氯苠基(2-氨基-1,3,4-噻二唑-5-基)二硫醚(3i)对 MCF-7 细胞表现了显著的抗 增殖活性 $\left(\mathrm{IC}_{50}\right.$ 值为 $\left.1.78 \mu \mathrm{mol} / \mathrm{L}\right)$, 且对 $\mathrm{A} 549$ 细胞展现了最好的抑制效果 $\left(\mathrm{IC}_{50}\right.$ 值为 $\left.4.04 \mu \mathrm{mol} / \mathrm{L}\right)$.
\end{abstract}

关键词 1,3,4-噻二唑; 二硫醚; 合成; 抗肿瘤活性

\section{Synthesis and Antitumor Activities of Disulfide Derivatives Containing 1,3,4-Thiadiazole Moiety}

\author{
Li, Sha Jing, Fen Fu, Xiaoyun Zhao, Jijun Wang, Xuefeng \\ Li, Baolin Liu, Yuming Chen, Baoquan* \\ (School of Chemistry and Chemical Engineering, Tianjin University of Technology, Tianjin 300384)
}

\begin{abstract}
Ten novel disulfide derivatives containing 1,3,4-thiadiazole moiety were synthesized from appropriate thiols, thiourea and 2-amino-5-mercapto-1,3,4-thiadiazole via two steps reactions. All compounds were characterized by IR, ${ }^{1} \mathrm{H}$ NMR, ESI-MS techniques and elemental analysis. The in vitro antiproliferative activities of target compounds against SMMC-7721 (human liver cancer cell), MCF-7 (human breast cancer cell) and A549 (human lung cancer cell) cells were evaluated by CCK- 8 assay. The bioassay results demonstrated that most of the tested compounds showed better antiproliferative activities, and exhibited stronger effects than positive control 5-fluorouracil (5-FU) against various cancer cell lines. Particularly, $n$-propyl (2-amino-1,3,4-thiadiazol-5-yl)disulfide (3b) and $n$-butyl (2-amino-1,3,4-thiadiazol-5-yl)disulfide (3d) displayed highly effective antiproliferative effects against SMMC-7721 cells with $\mathrm{IC}_{50}$ values of 1.68 and $1.93 \mu \mathrm{mol} / \mathrm{L}$. 4-Chlorobenzyl (2-amino-1,3,4-thiadiazol-5-yl)disulfide (3i) exhibited significant antiproliferative activities against MCF-7 cells with $\mathrm{IC}_{50}$ value of $1.78 \mu \mathrm{mol} / \mathrm{L}$, and revealed the best inhibitory effect against $\mathrm{A} 549$ cells with $\mathrm{IC}_{50}$ value of $4.04 \mu \mathrm{mol} / \mathrm{L}$.
\end{abstract}

Keywords 1,3,4-thiadiazole; disulfide; synthesis; antitumor activity

恶性肿瘤是严重威胁人类健康的疾病之一，因此， 寻找新的治疗手段和靶标日益受到人们的关注. 硫氧还 蛋白(thioredoxin, Trx) 是一种普遍表达于各种生物组织 器官的小分子多功能蛋白质. Trx、硫氧还蛋白还原酶 (thioredoxin reductase, TrxR)和烟酰腺嘌呤二核苷磷酸 $(\mathrm{NADPH})$ 共同组成了 $\mathrm{Trx}$ 系统. 研究显示, Trx 系统和
肿瘤的关系非常密切, 影响着肿瘤的发生、发展、增殖、 分化的过程，甚至可以作为临床预后的一项指标. Trx 已 成为国际上肿瘤分子生物学研究中的一大热点, 是一个 极具临床意义的肿瘤治疗新靶点 ${ }^{[1 \sim 6]}$. 目前国际上进入 临床研究的硫氧还蛋白系统的靶向抗癌药物只有三 种 ${ }^{[6 \sim 8]}$ : 加拿大 Pharmacyclics Inc 研发的 Motexafin

* E-mail: chenbaoquan66@126.com

Received May 16, 2015; revised July 12, 2015; published online August 31, 2015

Project supported by the National Natural Science Foundation of China (No. 20971097) and the Tianjin Municipal Natural Science Foundation (No. 13JCYBJC24500).

国家自然科学基金(No. 20971097)和天津市自然科学基金(No. 13JCYBJC24500)资助项目. 
gadolinium, 是一种具有卟啉环类似结构的抗癌药物, 目前正在进行III期临床研究; 美国 ProlX Pharmaceuticals 公司研发的 PX-12, 是一种具有二硫醚结构的抗癌 药物, 目前正在进行 II 期临床研究; 以及北京大学研究 开发的乙烷硒啉(Ethaselen), 是一种具有苯并异硒唑酮 结构的抗癌药物, 目前已完成 I 期临床研究. 其中, PX-12 化学名称为 1-甲基丙基-2-咪唑基二硫醚, 是二硫 醚类抗癌化合物的代表分子, 也是世界上第一个进入临 床研究的二硫醚类抗癌化合物 ${ }^{[9,10]}$. 研究表明, PX-12 中 的二硫键是该药物分子与硫氧还蛋白还原酶和硫氧还 蛋白发生反应的关键活性区域. 其可以导致硫氧还蛋白 还原酶和硫氧还蛋白的活性下降而触发了其分子靶向 性的抗癌作用 ${ }^{[11,12]}$.

1,3,4-噻二唑是设计生物活性剂的重要先导分子, 具有抗癌 ${ }^{[13 \sim 19]}$ 、抗氧化 ${ }^{[16,20]}$ 、抗菌 ${ }^{[21 ~ 23]}$ 、抗抑郁 ${ }^{[24,25]}$ 、 抗真菌 ${ }^{[26,27]}$ 、抗癫㾁 ${ }^{[28,29]}$ 以及抗炎 ${ }^{[30,31]}$ 等多种生物活 性. 特别是 1,3,4-塞二唑衍生物的 “碳氮硫” 结构能作 为活性中心螯合生物体内的某些金属离子, 具有较好的 细胞组织通透性, 能够更好地发挥药效. 而且将不同生 物活性基团引入到 1,3,4-噻二唑母核上, 会对其生物活 性产生影响, 因此, 已成为唑类化合物研究的热 点 $^{[21,22,26,27,32]}$.

基于 1,3,4-噻二唑和二硫醚药效基团的生物活性特 征, 为了得到活性更好的抗肿瘤先导化合物, 合成了 10 个未见文献报道的含 1,3,4-噻二唑的二硫醚衍生物, 结 构经 IR, 1H NMR, ESI-MS 和元素分析测试确定, 并采 用 CCK-8 法测试目标化合物对肝癌细胞 SMMC-7721、 乳腺癌细胞 MCF-7 和肺癌细胞 A549等肿瘤细胞的增殖 抑制活性. 其合成路线见 Scheme 1.

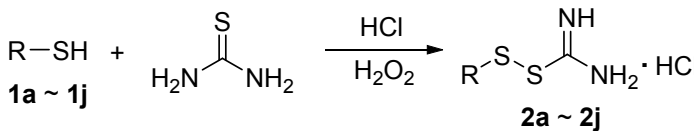

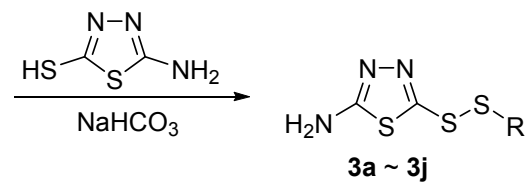

$\mathrm{R}=\mathrm{CH}_{2} \mathrm{CH}_{3}(\mathbf{a}), \mathrm{CH}_{2} \mathrm{CH}_{2} \mathrm{CH}_{3}(\mathbf{b}), \mathrm{CH}\left(\mathrm{CH}_{3}\right)_{2}(\mathbf{c}), \mathrm{CH}_{2}\left(\mathrm{CH}_{2}\right)_{2} \mathrm{CH}_{3}$ (d), $\mathrm{CH}_{3} \mathrm{CHCH}_{2} \mathrm{CH}_{3}(\mathbf{e}), \mathrm{CH}_{2}\left(\mathrm{CH}_{2}\right)_{3} \mathrm{CH}_{3}(\mathbf{f}), \mathrm{CH}_{2}\left(\mathrm{CH}_{2}\right)_{4} \mathrm{CH}_{3}(\mathbf{g}), \mathrm{PhCH}_{2}$ (h), 4- $\mathrm{ClC}_{6} \mathrm{H}_{4} \mathrm{CH}_{2}$ (i), 4- $\mathrm{CH}_{3} \mathrm{OC}_{6} \mathrm{H}_{4} \mathrm{CH}_{2}$ (j)

图式 1 目标化合物 3 的合成

Scheme 1 Synthetic route of the target compounds 3

\section{1 结果与讨论}

\section{1 目标化合物的合成}

合成不对称二硫醚的常用方法有硫醇与 Bunte 盐 (S-烃基硫代硫酸盐)反应 ${ }^{[33]}$ 、烃代硫基氯与硫醇(或硫
酚)反应以及二硫醚交换 ${ }^{[34]}$ 等. 我们参照相关文献[35], 以烷基硫醇和硫腿为原料, 经过氧化氢氧化生成烷基硫 代异硫脲盐酸盐, 不经处理, 在碱性条件下直接与 2-氨 基-5-颈基-1,3,4-噻二唑反应，合成了二硫梄化合物. 该 方法原料易得，操作简便，收率稳定.

\section{2 目标化合物的波谱分析}

红外光谱中, 目标化合物均在 $3400 \mathrm{~cm}^{-1}$ 附近出现 了氨基特征吸收峰, 并在 $1600 \mathrm{~cm}^{-1}$ 左右出现 $\mathrm{C}=\mathrm{N}$ 伸 缩振动吸收峰. 上述红外光谱特征峰的出现, 证明与目 标化合物的结构相吻合.

目标化合物(以 $3 \mathbf{a}$ 为例)核磁共振氢谱表明, 在 $\delta$ 7.53 位有一单峰, 为与噻二唑相连的氨基质子吸收峰. $\delta$ 2.92 位有一四重峰, 偶合常数为 $7.2 \mathrm{~Hz}$, 为 $\mathrm{SCH}_{2}$ 质子吸 收峰. $\delta 1.31$ 位有一三重峰, 偶合常数为 $7.2 \mathrm{~Hz}$, 为 $\mathrm{CH}_{3}$ 质子吸收峰. 核磁共振氢谱体现了目标化合物 3a 质子 吸收峰特征. ESI-MS 谱中出现了准分子离子峰 194.3 $[\mathrm{M}+\mathrm{H}]^{+}$, 表明其分子量为 193.3, 与其结构相符合.

\section{3 体外抗肿瘤活性}

以 5-氟尿嘧啶(5-FU)为阳性对照药, 用 CCK-8 法评 价了目标化合物 $\mathbf{3 a} \sim \mathbf{3 j}$ 对人体肝癌细胞 SMMC-7721, 乳腺癌细胞 MCF-7 和肺癌细胞 A549 等肿瘤细胞的增殖 抑制活性. 化合物的半数抑制浓度 $\left(\mathrm{IC}_{50}\right)$ 见表 1 .

\section{表 1 目标化合物的体外抗肿瘤活性}

Table 1 In vitro antitumor activities of target compounds against various cell lines

\begin{tabular}{cccc}
\hline \multirow{2}{*}{ Compd. } & \multicolumn{3}{c}{$\mathrm{IC}_{50}{ }^{a} /\left(\mu \mathrm{mol} \cdot \mathrm{L}^{-1}\right)$} \\
\cline { 2 - 4 } & $\mathrm{SMMC}-7721$ & $\mathrm{MCF}-7$ & $\mathrm{~A} 549$ \\
\hline $\mathbf{3 a}$ & 7.75 & 3.03 & 8.00 \\
$\mathbf{3 b}$ & 1.68 & 3.69 & 6.39 \\
$\mathbf{3 c}$ & 3.18 & 3.68 & 5.64 \\
$\mathbf{3 d}$ & 1.93 & 2.68 & 5.64 \\
$\mathbf{3 e}$ & 2.93 & 2.93 & 6.89 \\
$\mathbf{3 f}$ & 3.19 & 4.49 & 4.29 \\
$\mathbf{3 g}$ & 7.65 & 4.29 & 8.40 \\
$\mathbf{3 h}$ & 8.15 & 2.84 & 26.55 \\
$\mathbf{3 i}$ & 32.92 & 1.78 & 4.04 \\
$\mathbf{3 j}$ & 3.02 & 3.19 & 4.34 \\
$5-\mathrm{FU}$ & 5.62 & 14.26 & 8.13 \\
\hline${ }_{\text {实验数据为 } 3 \text { 次平行实验平均值. }}$
\end{tabular}

体外抗肿瘤活性测试结果表明, 对于不同的肿瘤细 胞, 大多数化合物表现了较好的增殖抑制活性. 对于 SMMC-7721 和 A549细胞，所有烷基取代的化合物 3a 3j 均比芐基取代的化合物 $3 \mathbf{h}$ 表现了更好的活性，而乙 基和正丁基取代的衍生物 3a 和 $3 \mathrm{~g}$ 比其它烷基取代的衍 生物表现了更弱的增殖抑制效果. 对于 SMMC-7721 细 胞, 多数化合物显示了较好的抗癌效果, 且活性优于阳 性对照药 5-氟尿嘧啶. 其中, 化合物 $\mathbf{3 b}$ 和 $\mathbf{3 d}$ 对 SMMC- 
7721 细胞显示了高效的增殖抑制活性, $\mathrm{IC}_{50}$ 值分别为 1.68 和 $1.93 \mu \mathrm{mol} / \mathrm{L}$. 与苄基取代的化合物 $3 \mathbf{h}$ 比较, 对氯 苄基取代的化合物 $3 \mathbf{i}$ 大大降低了抗癌活性, 而甲氧基苠 基取代的化合物 $\mathbf{3 j}$ 表现了较好的抗癌效果. 对于 MCF-7 细胞, 所有化合物均表现了较好的生物活性, 且 活性优于 5-氟尿嘧啶, 特别是化合物 3i 对 MCF-7 细胞 表现了显著的增殖抑制活性, $\mathrm{IC}_{50}$ 值为 $1.78 \mu \mathrm{mol} / \mathrm{L}$, 但 烷基链长短以及芳基苯环上的取代基对抗癌活性没有 产生显著影响, 没有体现明显的规律性. 对于 A549 细 胞, 除茮基取代化合物 $\mathbf{3 h}$ 外 $\left(\mathrm{IC}_{50}=26.55 \mu \mathrm{mol} / \mathrm{L}\right)$, 其它 化合物都显示了较好的生物活性, 多数化合物的活性优 于对照药 5-氟尿嘧啶. 其中, 对氯芳基取代的化合物 3i 对 A549 细胞显示了最好的抑制效果, $\mathrm{IC}_{50}$ 值为 4.04 $\mu \mathrm{mol} / \mathrm{L}$. 与化合物 $\mathbf{3 h}$ 比较, 氯原子和甲氧基基团的引入 均大大增强了化合物的抗癌活性, 但化合物 $\mathbf{3 i}$ 抗癌效果 稍强于化合物 $\mathbf{3} \mathbf{j}$, 没有表现出显著差异. 上述结果对通 过结构改造进一步改善化合物的生物活性奠定了基础.

\section{2 结论}

合成了 10 个新的含 1,3,4-噻二唑的二硫醚衍生物, 并利用 IR, ${ }^{1} \mathrm{H}$ NMR, ESI-MS 和元素分析对目标化合物 进行了结构表征. 采用 CCK-8 法测试了目标产物对人 体肝癌细胞 SMMC-7721、乳腺癌细胞 MCF-7 和肺癌细 胞 A549 等肿瘤细胞的增殖抑制活性. 测试结果表明, 对于不同的肿瘤细胞, 大多数试验化合物显示了较好的 增殖抑制活性, 且其活性优于阳性对照药 5-氟尿嘧啶. 尤其是化合物 3b 和 3d, 对 SMMC-7721 细胞显示了高 效的增殖抑制效果, $\mathrm{IC}_{50}$ 值分别为 1.68 和 $1.93 \mu \mathrm{mol} / \mathrm{L}$. 化合物 3i 对 MCF-7 细胞表现了显著的抗增殖活性, $\mathrm{IC}_{50}$ 值为 $1.78 \mu \mathrm{mol} / \mathrm{L}$, 且对 A549 细胞展现了最好的抑制效 果, $\mathrm{IC}_{50}$ 值为 $4.04 \mu \mathrm{mol} / \mathrm{L}$. 目前进一步的结构优化和构 效关系的深入研究正在进行中.

\section{3 实验部分}

\section{1 仪器与试剂}

熔点用 X-4 数字显示显微熔点仪测定(温度未经校 正); 红外光谱仪为 Avartar370 型( $\mathrm{KBr}$ 压片); 核磁共振 仪为 Bruker Avance III $400 \mathrm{MHz}$ 型(DMSO- $d_{6}$ 为溶剂, TMS 为内标); 质谱仪为 Xevo G2 QTof 型(ESI-MS); 元 素分析仪为 Flash EA1112 型; 酶标仪为 BioTek Epoch 型; CCK-8 试剂盒, 日本同仁化学公司产品; 肝癌细胞 SMMC-7721, 乳腺癌细胞 MCF-7 和肺癌细胞 A549 购于 中国医学科学院肿瘤细胞库. 所用试剂均为国产市售分 析纯或化学纯, 部分有机试剂使用前经常规处理.

\section{2 实验方法}

3.2 .1 烷基(2-氨基-1,3,4-噻二唑-5-基)二硫醚 $(3 \mathbf{a} \sim$ $3 \mathbf{j}$ ) 的合成

在 $100 \mathrm{~mL}$ 三口瓶中, 加入 $0.76 \mathrm{~g}(10 \mathrm{mmol})$ 硫脲, 13 $\mathrm{mmol}$ 硫醇, $5 \mathrm{~mL}$ 水和 $15 \mathrm{~mL}$ 乙醇, 搅拌使其溶解. 在 $0 \sim 5{ }^{\circ} \mathrm{C}$ 下, 加入 $1.1 \mathrm{~mL}$ 浓盐酸, 搅拌下滴入 $1.24 \mathrm{~g}(11$ mmol) $30 \%$ 过氧化氢水溶液(约 $30 \mathrm{~min}$ 滴毕), 继续摚拌 反应 $3 \mathrm{~h}$, 减压蒸去溶剂, 得取代統基异硫脲盐酸盐, 直 接用于下一步反应.

向上述反应器中, 加入 $1.33 \mathrm{~g}(10 \mathrm{mmol}) 2$-氨基-5颈基-1,3,4-噻二唑和 $20 \mathrm{~mL}$ 乙醇, 室温搅拌下, 缓慢滴 加含 $1.26 \mathrm{~g}$ (15 mmol)碳酸氢钠的水 $(20 \mathrm{~mL}$ ) 溶液(约 20 $\min$ 滴毕), 室温继续搅拌反应 $2 \mathrm{~h}$, 减压蒸去大部分溶 剂, 析出固体, 抽滤, 滤饼以乙醇-水重结晶, 得纯品 $\mathbf{3 a} \sim \mathbf{3 j}$.

乙基(2-氨基-1,3,4-噻二唑-5-基)二硫醚(3a)：白色固 体, 收率 82.5\%. m.p. $228.8 \sim 230.7{ }^{\circ} \mathrm{C} ;{ }^{1} \mathrm{H}$ NMR $\left(\right.$ DMSO$\left._{-}, 400 \mathrm{MHz}\right) \delta: 7.53\left(\mathrm{~s}, 2 \mathrm{H}, \mathrm{NH}_{2}\right), 2.92$ (q, $J=$ $\left.7.2 \mathrm{~Hz}, 2 \mathrm{H}, \mathrm{SCH}_{2}\right), 1.31\left(\mathrm{t}, J=7.2 \mathrm{~Hz}, 3 \mathrm{H}, \mathrm{CH}_{3}\right)$; IR (KBr) $v: 3416,2963,1617,1504,1134,618 \mathrm{~cm}^{-1}$; ESI-MS m/z: $194.3[\mathrm{M}+\mathrm{H}]^{+}$. Anal. calcd for $\mathrm{C}_{4} \mathrm{H}_{7} \mathrm{~N}_{3} \mathrm{~S}_{3}: \mathrm{C} 24.85, \mathrm{H}$ 3.65, N 21.74; found C 24.76, H 3.62, N 21.80.

正丙基(2-氨基-1,3,4-噻二唑-5-基)二硫醚(3b): 白 色固体，收率 78.7\%. m.p. 174.1 175.5 ${ }^{\circ} \mathrm{C}$; ${ }^{1} \mathrm{H}$ NMR $\left(\mathrm{DMSO}-d_{6}, 400 \mathrm{MHz}\right) \delta: 7.53\left(\mathrm{~s}, 2 \mathrm{H}, \mathrm{NH}_{2}\right), 2.90(\mathrm{t}, J=7.2$ $\left.\mathrm{Hz}, 2 \mathrm{H}, \mathrm{SCH}_{2}\right), 1.66 \sim 1.75\left(\mathrm{~m}, 2 \mathrm{H}, \mathrm{CH}_{2}\right), 0.94(\mathrm{t}, J=7.2$ $\mathrm{Hz}, 3 \mathrm{H}, \mathrm{CH}_{3}$ ); IR (KBr) v: 3417, 2961, 1619, 1504, 1135, $619 \mathrm{~cm}^{-1}$; ESI-MS m/z: $208.3[\mathrm{M}+\mathrm{H}]^{+}$. Anal. calcd for $\mathrm{C}_{5} \mathrm{H}_{9} \mathrm{~N}_{3} \mathrm{~S}_{3}$ : C 28.96, H 4.38, N 20.27; found C 28.88, H $4.41, \mathrm{~N} 20.33$.

异丙基(2-氨基-1,3,4-噻二唑-5-基)二硫醚(3c)：白色 固体，收率 79.5\%. m.p. $177.8 \sim 179.5{ }^{\circ} \mathrm{C} ;{ }^{1} \mathrm{H}$ NMR $\left(\mathrm{DMSO}-d_{6}, 400 \mathrm{MHz}\right) \delta: 7.50\left(\mathrm{~s}, 2 \mathrm{H}, \mathrm{NH}_{2}\right), 3.22 \sim 3.33$ (m, 1H, SCH), 1.31 (d, $J=6.8 \mathrm{~Hz}, 6 \mathrm{H}, \mathrm{CH}_{3}$ ); IR (KBr) v: 3416, 2962, 1618, 1505, 1137, $619 \mathrm{~cm}^{-1}$; ESI-MS m/z: $208.3[\mathrm{M}+\mathrm{H}]^{+}$. Anal. calcd for $\mathrm{C}_{5} \mathrm{H}_{9} \mathrm{~N}_{3} \mathrm{~S}_{3}: \mathrm{C} 28.96, \mathrm{H}$ 4.38, N 20.27; found C 29.02, H 4.34, N 20.34.

正丁基(2-氨基-1,3,4-噻二唑-5-基)二硫醚(3d)：白 色固体，收率 77.8\%. m.p. 166.7 168.2 ${ }^{\circ} \mathrm{C} ;{ }^{1} \mathrm{H}$ NMR $\left(\mathrm{DMSO}-d_{6}, 400 \mathrm{MHz}\right) \delta: 7.53\left(\mathrm{~s}, 2 \mathrm{H}, \mathrm{NH}_{2}\right), 2.92(\mathrm{t}, J=7.2$ $\left.\mathrm{Hz}, 2 \mathrm{H}, \mathrm{SCH}_{2}\right), 1.62 \sim 1.70\left(\mathrm{~m}, 2 \mathrm{H}, \mathrm{CH}_{2}\right), 1.32 \sim 1.41(\mathrm{~m}$, $\left.2 \mathrm{H}, \mathrm{CH}_{2}\right), 0.88\left(\mathrm{t}, J=7.2 \mathrm{~Hz}, 3 \mathrm{H}, \mathrm{CH}_{3}\right)$; IR (KBr) v: 3416, 2956, 1618, 1502, 1134, $620 \mathrm{~cm}^{-1}$; ESI-MS m/z: 222.4 $[\mathrm{M}+\mathrm{H}]^{+}$. Anal. calcd for $\mathrm{C}_{6} \mathrm{H}_{11} \mathrm{~N}_{3} \mathrm{~S}_{3}$ : C 32.55, H 5.01, N 18.98; found C 32.64, H 4.99, N 19.04 . 
2-丁基(2-氨基-1,3,4-噻二夾-5-基)二硫醚(3e)：白色 固体, 收率 $76.9 \%$. m.p. $140.5 \sim 142.6{ }^{\circ} \mathrm{C} ;{ }^{1} \mathrm{H}$ NMR (DMSO- $\left.d_{6}, 400 \mathrm{MHz}\right) \delta: 7.50\left(\mathrm{~s}, 2 \mathrm{H}, \mathrm{NH}_{2}\right.$ ), $3.01 \sim 3.10$ $(\mathrm{m}, 1 \mathrm{H}, \mathrm{SCH}), 1.52 \sim 1.62\left(\mathrm{~m}, 1 \mathrm{H}, \mathrm{CH}_{2}-\mathrm{H}_{\mathrm{a}}\right), 1.62 \sim 1.73$ $\left(\mathrm{m}, 1 \mathrm{H}, \mathrm{CH}_{2}-\mathrm{H}_{\mathrm{a}}\right), 1.30\left(\mathrm{~d}, J=6.8 \mathrm{~Hz}, 3 \mathrm{H}, \mathrm{CH}_{3}\right), 0.91(\mathrm{t}$, $J=7.2 \mathrm{~Hz}, 3 \mathrm{H}, \mathrm{CH}_{3}$ ); IR (KBr) v: 3416, 2966, 1618, 1512, $1134,619 \mathrm{~cm}^{-1}$; ESI-MS m/z: $222.4[\mathrm{M}+\mathrm{H}]^{+}$. Anal. calcd for $\mathrm{C}_{6} \mathrm{H}_{11} \mathrm{~N}_{3} \mathrm{~S}_{3}$ : C 32.55, H 5.01, N 18.98; found C 32.60, H 5.04, N 18.92 .

正戊基(2-氨基-1,3,4-塞二唑-5-基)二硫醚(3f)：浅黄 色固体, 收率 85.6\%. m.p. 236.2 237.9 ${ }^{\circ} \mathrm{C} ;{ }^{1} \mathrm{H}$ NMR (DMSO- $\left.d_{6}, 400 \mathrm{MHz}\right) \delta: 7.52\left(\mathrm{~s}, 2 \mathrm{H}, \mathrm{NH}_{2}\right), 2.92$ (t, $J=7.2$ $\left.\mathrm{Hz}, 2 \mathrm{H}, \mathrm{SCH}_{2}\right), 1.65 \sim 1.72\left(\mathrm{~m}, 2 \mathrm{H}, \mathrm{CH}_{2}\right), 1.30 \sim 1.33(\mathrm{~m}$, $\left.4 \mathrm{H}, \mathrm{CH}_{2}\right), 0.86\left(\mathrm{t}, J=7.2 \mathrm{~Hz}, 3 \mathrm{H}, \mathrm{CH}_{3}\right)$; IR (KBr) $v: 3443$, 3134, 1636, 1502, 1133, 1108, $615 \mathrm{~cm}^{-1}$; ESI-MS $\mathrm{m} / \mathrm{z}$ : $236.4[\mathrm{M}+\mathrm{H}]^{+}$. Anal. calcd for $\mathrm{C}_{7} \mathrm{H}_{13} \mathrm{~N}_{3} \mathrm{~S}_{3}: \mathrm{C} 35.72, \mathrm{H}$ 5.57, N 17.85; found C 35.61, H 5.52, N 17.91.

正己基(2-氨基-1,3,4-噻二唑-5-基)二硫醚(3g): 浅黄 色固体, 收率 80.3\%. m.p. $232.5 \sim 234.4{ }^{\circ} \mathrm{C}$; IR (KBr) v: $3444,3145,1636,1505,1132,1108,616 \mathrm{~cm}^{-1} ;{ }^{1} \mathrm{H}$ NMR (DMSO- $\left.d_{6}, 400 \mathrm{MHz}\right) \delta: 7.50\left(\mathrm{~s}, 2 \mathrm{H}, \mathrm{NH}_{2}\right), 2.92(\mathrm{t}$, $\left.J=7.2 \mathrm{~Hz}, 2 \mathrm{H}, \mathrm{SCH}_{2}\right), 1.64 \sim 1.71\left(\mathrm{~m}, 2 \mathrm{H}, \mathrm{CH}_{2}\right), 1.32 \sim$ $1.38\left(\mathrm{~m}, 2 \mathrm{H}, \mathrm{CH}_{2}\right), 1.23 \sim 1.29\left(\mathrm{~m}, 4 \mathrm{H}, \mathrm{CH}_{2}\right), 0.86(\mathrm{t}, J=$ $7.2 \mathrm{~Hz}, 3 \mathrm{H}, \mathrm{CH}_{3}$ ); ESI-MS m/z: $250.4[\mathrm{M}+\mathrm{H}]^{+}$. Anal. calcd for $\mathrm{C}_{8} \mathrm{H}_{15} \mathrm{~N}_{3} \mathrm{~S}_{3}$ : C 38.52, H 6.06, N 16.85; found $\mathrm{C}$ 38.62, H 6.10, N 16.78.

芐基(2-氨基-1,3,4-噻二唑-5-基)二硫醚(3h): 浅黄 色固体, 收率 60.1\%. m.p. 198.2 200.2 ${ }^{\circ} \mathrm{C} ;{ }^{1} \mathrm{H}$ NMR $\left(\right.$ DMSO- $\left.d_{6}, 400 \mathrm{MHz}\right) \delta: 7.50\left(\mathrm{~s}, 2 \mathrm{H}, \mathrm{NH}_{2}\right), 7.27 \sim 7.35$ (m, 5H, ArH), 4.17 (s, 2H, $\mathrm{SCH}_{2}$ ); IR (KBr) v: 3451, 3134, 2361, 2074, 1637, 1506, 1401, 1193, 1113, $620 \mathrm{~cm}^{-1}$; ESI-MS $m / z: 256.4[\mathrm{M}+\mathrm{H}]^{+}$. Anal. calcd for $\mathrm{C}_{9} \mathrm{H}_{9} \mathrm{~N}_{3} \mathrm{~S}_{3}: \mathrm{C}$ 42.33, H 3.55, N 16.45; found C 42.45, H 3.59, N 16.39.

4-氯苄基(2-氨基-1,3,4-噻二唑-5-基)二硫醚 (3i): 白 色固体, 收率 58.6\%. m.p. 197.5.2 199.2 ${ }^{\circ} \mathrm{C} ;{ }^{1} \mathrm{H}$ NMR (DMSO- $\left.d_{6}, 400 \mathrm{MHz}\right) \delta: 7.52$ (s, 2H, NH $\left.\mathrm{N}_{2}\right), 7.39$ (d, $J=$ $7.2 \mathrm{~Hz}, 2 \mathrm{H}, \mathrm{ArH}$ ) , 7.31 (d, J=8.4 Hz, 2H, ArH ), 4.16 (s, $\left.2 \mathrm{H}, \mathrm{SCH}_{2}\right)$; IR (KBr) v: 3449, 3160, 2360, 2075, 1635, 1508, 1401, 1113, $619 \mathrm{~cm}^{-1}$; ESI-MS m/z: $290.3[\mathrm{M}+\mathrm{H}]^{+}$. Anal. calcd for $\mathrm{C}_{9} \mathrm{H}_{8} \mathrm{ClN}_{3} \mathrm{~S}_{3}$ : C 37.30, H 2.78, N 14.50; found C 37.37, H 2.81, N 14.45.

4-甲氧苄基(2-氨基-1,3,4-噻二唑-5-基)二硫醚 $(\mathbf{3} \mathbf{j})$ : 白色固体, 收率 62.3\%. m.p. $158.9 \sim 161.2{ }^{\circ} \mathrm{C} ;{ }^{1} \mathrm{H}$ NMR (DMSO- $\left.d_{6}, 400 \mathrm{MHz}\right) \delta: 7.51\left(\mathrm{~s}, 2 \mathrm{H}, \mathrm{NH}_{2}\right), 7.27$ (d, $J=$ $8.8 \mathrm{~Hz}, 2 \mathrm{H}, \mathrm{ArH}), 6.91$ (d, J=8.4 Hz, 2H, ArH ), 4.13 (s, $\left.2 \mathrm{H}, \mathrm{SCH}_{2}\right), 3.75\left(\mathrm{~s}, 3 \mathrm{H}, \mathrm{OCH}_{3}\right)$; IR ( $\left.\mathrm{KBr}\right) v: 3421,3156$, 2361, 2072, 1632, 1514, 1385, 1072, $619 \mathrm{~cm}^{-1}$; ESI-MS $m / z: 286.4[\mathrm{M}+\mathrm{H}]^{+}$. Anal. calcd for $\mathrm{C}_{10} \mathrm{H}_{11} \mathrm{~N}_{3}-\mathrm{OS}_{3}: \mathrm{C}$ 42.08, H 3.88, N 14.72; found C 42.19, H 3.84, N 14.66.

3.2 .2 抗肿瘤活性测试

采用 CCK-8 试剂盒, 按照试剂盒说明书进行测 定. 具体操作如下：将 5-氟尿嘧啶及合成的 9 个新化合 物用 DMSO 配成 $1.0 \times 10^{-2} \mathrm{~mol} / \mathrm{L}$ 浓度的储备液, 实验 时用细胞培养液 RPMI-1640 稀释到所需浓度. 将处于指 数生长期细胞用 $0.25 \%$ 胰酶消化, 调节细胞浓度为 $5 \times 10^{4}$ 细胞 $/ \mathrm{mL}$ ，以 $200 \mu \mathrm{L} /$ 孔接种于 96 孔培养板中(即 $10^{4}$ 细胞/ 孔), 置 $37{ }^{\circ} \mathrm{C}, 5 \% \mathrm{CO}_{2}$ 培养箱中培养 $24 \mathrm{~h}$. 倒 出培养基后加入新鲜培养基 $199 \mu \mathrm{L} /$ 的及不同浓度的化 合物 $1 \mu \mathrm{L} /$ 孔，使其终浓度为 $1.0 \times 10^{-4} 、 0.5 \times 10^{-4}$ 、 $1.0 \times 10^{-5} 、 0.5 \times 10^{-5} 、 1.0 \times 10^{-6} \mathrm{~mol} / \mathrm{L}$, 每个浓度设 3 个平行样, $37{ }^{\circ} \mathrm{C}, 5 \% \mathrm{CO}_{2}$ 培养箱中继续培养 $48 \mathrm{~h}$. 倒出 培养基, 用 $\mathrm{PBS}$ 洗 3 次, 加入新鲜培养基 $90 \mu \mathrm{L}$ 及 CCK-8 $10 \mu \mathrm{L}$, 于 $37{ }^{\circ} \mathrm{C}, 5 \% \mathrm{CO}_{2}$ 培养箱中培养 $1 \mathrm{~h}$, 将 96 孔板放入酶标仪样品池中振荡 $30 \mathrm{~s}$, 使样品混合均 匀, 设置 6 个空白组(培养基、CCK-8、DMSO), 5 个对 照组(细胞、培养基、CCK-8、DMSO)，在酶标仪 $450 \mathrm{~nm}$ 读板, 测出吸光度值(OD 值). 按公式: 相对细胞存活 率 $=$ (供试组 OD 值一空白 OD 值)/(对照组 OD 值一空白 OD 值) $\times 100 \%$, 细胞抑制率 $=1$-相对细胞存活率, 计 算各组细胞生长抑制率，绘制剂量与效应曲线. 用作图 法计算系列化合物对 4 种癌细胞株的半数抑制率浓度 $\mathrm{IC}_{50}$ 值.

辅助材料(Supporting Information) 所合成化合物的 ${ }^{1} \mathrm{H}$ NMR 谱图. 这些材料可以免费从本刊网站(http:// sioc-journal.cn/)上下载.

\section{References}

[1] Arnér, E. S. J.; Holmgren, A. Eur. J. Biochem. 2000, 267, 6102.

[2] Kirkpatrick, D. L.; Kuperus, M.; Dowdeswell, M.; Potier, N.; Donald, L. J.; Kunkel, M.; Berggren, M.; Angulo, M.; Powis, G. Biochem. Pharmacol. 1998, 55, 987.

[3] Tan, Q.; Li, J.; Yin, H.-W.; Wang, L.-H.; Tang, W.-C.; Zhao, F.; Liu, X.-M.; Zeng, H.-H. Invest New Drugs 2010, 28, 205.

[4] Xing, F.-X.; Li, S.-L.; Ge, X.-Y.; Wang, C.-Y.; Zeng, H.-H.; Li, D.; Dong, L. Oral Oncol. 2008, 44, 963.

[5] Urig, S.; Becker, K. Semin. Cancer Biol. 2006, 16, 452.

[6] He, J.; Li, D.-D.; Xiong, K.; Ge, Y.-J.; Jin, H.-W.; Zhang, G.-Z.; Hong, M.-S.; Tian, Y.-L.; Yin, J.; Zeng, H.-H. Bioorg. Med. Chem. 2012, 20, 3816.

[7] Li, D.-D.; Tao, Z.-W. Tianjin Pharm. 2014, 26, 64 (in Chinese). (李冬冬, 陶遵威, 天津药学, 2014, 26, 64.)

[8] Lin, T. S.; Naumovski, L.; Lecane, P. S.; Lucas, M. S.; Moran, M. E.; Cheney, C.; Lucas, D. M.; Phan, S. C.; Miller, R. A.; Byrd, J. C. Leukemia Lymphoma 2009, 50, 1977. 
[9] Ramanathan, R. K.; Abbruzzese, J.; Dragovich, T.; Kirkpatrick, L.; Guillen, J. M.; Baker, A. F.; Pestano, L. A.; Green, S.; Von Hoff, D. D. Cancer Chemother. Pharmacol. 2011, 67, 503.

[10] Baker, A. F.; Adab, K. N.; Raghunand, N.; Chow, H. H. S.; Stratton, S. P.; Squire, S. W.; Boice, M.; Pestano, L. A.; Kirkpatrick, D. L.; Dragovich, T. Invest New Drugs 2013, 31, 631.

[11] You, B. R.; Shin, H. R.; Park, W. H. Int. J. Oncol. 2014, 44, 301.

[12] Shin, H. R.; You, B. R.; Park, W. H. Oncol. Lett. 2013, 6, 1804.

[13] Li, Y.-J.; Yu, Y.; Jin, K.; Gao, L.-X.; Luo, T.-C.; Sheng. L.; Shao, X.; Li, J. Chin. J. Org. Chem. 2015, 35, 129 (in Chinese). (李英俊, 于洋, 靳焜, 高立信, 罗潼川, 盛丽, 邵昕, 李佳, 有 机化学, 2015, 35, 129.)

[14] Li, Y.-J.; Luo, T.-C.; Jin, K.; Gao, L.-X.; Shao, X.; Sheng. L.; Yu, Y.; Li, J. Chin. J. Org. Chem. 2014, 34, 325 (in Chinese). (李英俊, 罗潼川, 靳焜, 高立信, 邵昕, 盛丽, 于洋, 李佳, 有 机化学, 2014, 34, 325.)

[15] Hosseinzadeh, L.; Khorand, A.; Aliabadi, A. Arch. Pharm. Chem. Life Sci. 2013, 346, 812.

[16] Hamama, W. S.; Gouda, M. A.; Badr, M. H.; Zoorob, H. H. Med. Chem. Res. 2013, 22, 3556.

[17] Zhao, Y.; Ouyang, G.-P.; Xu, W.-M.; Jin, L.-H.; Yuan, K. Chin. J. Org. Chem. 2010, 30, 1093 (in Chinese).

(赵云, 欧阳贵平, 徐维明, 金林红, 袁凯, 有机化学, 2010, 30, 1093.)

[18] Abdo, N. Y. M.; Kamel, M. M. Chem. Pharm. Bull. 2015, 63, 369.

[19] Yadagiri, B.; Gurrala, S.; Bantu, R.; Nagarapu, L.; Polepalli, S.; Srujana, G.; Jain, N. Bioorg. Med. Chem. Lett. 2015, 25, 2220.

[20] Khan, I.; Ali, S.; Hameed, S.; Rama, N. H.; Hussain, M. T.; Wadood, A.; Uddin, R.; Ul-Had, Z.; Khan, A.; Ali, S.; Choudhary, M. Z. Eur. J. Med. Chem. 2010, 45, 5200.

[21] Wang, S.-X.; Zuo, L.; Fan, Z.-J.; Zhang, Z.-C.; Zhang, J.-F.; Xiong, L.-X.; Fu, Y.-F.; Fang, Z.; Wu, Q.-J.; Zhang, Y.-J. Chin. J. Org. Chem. 2013, 33, 2367 (in Chinese).
(王守信, 左翔, 范志金, 张正财, 张聚方, 熊丽霞, 付一峰, 房 震, 吴青君, 张友军, 有机化学, 2013, 33, 2367.)

[22] Patel, H.; Mishra, L.; Noolvi, M.; Karpoormath, R.; Cameotra, S. S. Arch. Pharm. Chem. Life Sci. 2014, 347, 668.

[23] Yao, M.-X.; An, Y.; Yan, J.; Tian, X.; Wei, S. Chin. J. Org. Chem. 2013, 33, 1015 (in Chinese).

(姚明星, 安悦, 间杰, 田星, 魏诗, 有机化学, 2013, 33, 1015.)

[24] Yusuf, M.; Khan, R. A.; Ahmed, B. Bioorg. Med. Chem. 2008, 16, 8029.

[25] Clerici, F.; Pocar, D. J. Med. Chem. 2001, 44, 931.

[26] Xu, W.-M.; Li, S.-Z.; He, M.; Yang, S.; Li, X.-Y.; Li, P. Bioorg. Med. Chem. Lett. 2013, 23, 5821.

[27] Güzeldemirci, N. U.; Şatana, D.; Küçükbasmac1, Ö. J. Enzyme Inhib. Med. Chem. 2013, 28, 968.

[28] Siddiqui, N.; Ahuja, P.; Malik, S.; Arya, S. K. Arch. Pharm. Chem. Life Sci. 2013, 346, 819.

[29] Harish, K. P.; Mohana, K. N.; Mallesha, L. Russian J. Bioorg. Chem. 2014, 40, 97.

[30] Dekhane, D. V.; Pawar, S. S.; Gupta, S.; Shingare, M. S.; Patil, C. R.; Thore, S. N. Bioorg. Med. Chem. Lett. 2011, 21, 6527.

[31] Amir, M.; Kumar, H.; Javed, S. A. Arch. Pharm. Chem. Life Sci. 2007, 340, 577.

[32] Zhao, H.-C.; Shi, Y.-P.; Liu, Y.-M.; Li, C.-W.; Xuan, L.-N.; Wang, P.; Zhang, K.; Chen, B.-Q. Bioorg. Med. Chem. Lett. 2013, 23, 6577.

[33] Gong, J.-X.; Sheng, L.; Yao, L.-G.; Li, J.; Zhou, Y.-B.; Guo, Y.-W. Chin. J. Org. Chem. 2012, 32, 593 (in Chinese).

(龚景旭, 盛丽, 姚励功, 李佳, 周宇波, 郭跃伟, 有机化学, 2012, 32, 593.)

[34] Han, M.; Lee, J. T.; Hahn, H. G. Tetrahedron Lett. 2011, 52, 236.

[35] Kirkpatrick, D. L.; Jimale, M. L.; King, K. M.; Chen, T. Eur. J. Med. Chem. 1992, 27, 33. 